Journal of the Operations Research

Society of Japan

Vol. 40, No. 1, March 1997

\title{
AN M/G/1 QUEUE WITH FINITE POPULATION AND GATED SERVICE DISCIPLINE
}

\author{
Christos Langaris Apostolos Katsaros \\ University of Ioannina
}

(Received November 8, 1995; Revised June 7, 1996)

\begin{abstract}
A queueing model with finite customer population (an $\mathrm{M} / \mathrm{G} / 1 / / \mathrm{N}$ model), multiple server vacations and gated service policy is considered. For such a model, the steady state probabilities and the customers' waiting time are analyzed. Numerical results for the mean waiting times are obtained and used to draw conclusions on the system performance and to compare the present model with the corresponding model with exhaustive service. The comparison shows that, under variations on the mean vacation period and on the offered load, the two models behave in completely different ways. This is more apparent in case of large vacation periods and seems to be a consequence of the vacation principle.
\end{abstract}

\section{Introduction-The model}

The present work has been motivated by a remark made in Takagi [2]. More precisely, in Takagi [2], an $\mathrm{M} / \mathrm{G} / 1$ queueing system with a finite population $\mathrm{N}$, multiple vacations and exhaustive service was studied. The author pointed out that "it will be challenging to extend the approach to those systems with gated or limited service policies".

Although almost all papers on queues with server vacations concern systems with an infinite population of customers, it is true that in all applications of these models, mainly in computer and communication networks, the number of customers involved is finite.

Our model here can be described as an $M / G / 1$ queue with a finite population $N$. There are a "source" and a single-server queue and each of the $\mathrm{N}$ customers is, at any time, either in the source or in the queue. A customer in the source arrives to the queue according to an exponential distribution with parameter $\lambda$ and is served according to a general distribution with probability density function (p.d.f.) $b(t)$, distribution function (D.F.) $B(t)$ and finite mean $\bar{b}$. The service policy is "gated", i.e., the server serves only the customers that he finds in the queue when he starts serving and after that he departs for a vacation. When the vacation is ended the server either starts serving again or, if the queue is empty, he repeates the vacation. We assume that the vacation time is also arbitrarily distributed with p.d.f. $v(t)$, D.F. $V(t)$ and finite mean $\bar{v}$. As we mentioned above, a similar model but with exhaustive service has been studied in Takagi [2], while Takine and Hasegawa [3] have studied an $\mathrm{M} / \mathrm{G} / 1$ model with multiple vacations and gated service but assuming an infinite customer population. For a full description of the different service policies, see Takagi [1].

For our model, we study in Section 2 the steady state probabilities, while a formula for the Laplace-Stieltjes transform (LST) of the customers' waiting time is obtained in Section 3. In Section 4 finally, we present some numerical results for the mean waiting time, for various values of the parameters, and use them to compare the model with the corresponding models with exhaustive service and with infinite customer population.

\section{System state probabilities}

Throughout this work, the customers found by the server in the queue upon commencing service, will be called "primary" customers, while the customers arriving during the service 
period and the following vacation time will be called "secondary" customers.

Let $n(t)$ be the number of primary customers at the beginning of the service period which is in progress at time t. We use $n(t)=0$ if the server is on vacation at time $\mathrm{t}$. Let also $A_{n(t)}$ be the specific number of the primary customer (out of the $n(t)$ ) who is in service at time $t$. Finally, denote by $m(t)$ the number of the secondary customers in the system at time t. Define now

$$
\begin{gathered}
p^{(k, m)}(r, x, t) d x=\operatorname{Pr}\left[n(t)=k, A_{n(t)}=m, m(t)=r, x<S(t) \leq x+d x\right], \\
q(r, x, t) d x=\operatorname{Pr}\left[n(t)=0, m(t)=r, x<V_{0}(t) \leq x+d x\right],
\end{gathered}
$$

where $S(t), V_{0}(t)$ is the elapsed service time of the customer in service or the elapsed vacation time at time $t$, respectively.

By connecting as usual the probabilities at time $t$ with the corresponding quantities at $t+d t$ and assuming steady state we arrive at

$$
\begin{array}{r}
\frac{d}{d x} p^{(k, m)}(r, x)+[\lambda(N-k-r+m-1)+\bar{s}(x)] p^{(k, m)}(r, x) \\
=\lambda(N-k-r+m) p^{(k, m)}(r-1, x), \\
\frac{d}{d x} q(r, x)+[\lambda(N-r)+\bar{u}(x)] q(r, x)=\lambda(N-r+1) q(r-1, x),
\end{array}
$$

where

$$
\bar{s}(x)=\frac{b(x)}{1-B(x)}, \quad \bar{u}(x)=\frac{v(x)}{1-V(x)} .
$$

and $p^{(k, m)}(r, x)=\lim _{t \rightarrow \infty} p^{(k, m)}(r, x, t), q(r, x)=\lim _{t \rightarrow \infty} q(r, x, t)$.

Equations (2.1) must be solved under the boundary conditions, $q(N, 0)=0$, and

$$
p^{(k, m)}(r, 0)=\int_{0}^{\infty} p^{(k, m-1)}(r, x) \bar{s}(x) d x, \quad 1<m \leq k,
$$

$$
p^{(k, 1)}(0,0)=\int_{0}^{\infty} q(k, x) \bar{u}(x) d x, \quad k \geq 1
$$

$$
q(n, 0)=\sum_{k=1}^{N} \int_{0}^{\infty} p^{(k, k)}(n, x) \bar{s}(x) d x, \quad 1 \leq n \leq N-1
$$

$$
q(0,0)=\sum_{k=1}^{N} \int_{0}^{\infty} p^{(k, k)}(0, x) \bar{s}(x) d x+\int_{0}^{\infty} q(0, x) \bar{u}(x) d x
$$

If now we define

$$
P^{(k, m)}(z, x)=\frac{\sum_{r=0}^{N-k+m-1} p^{(k, m)}(r, x) z^{N-k+m-r-1}}{1-B(x)}, \quad Q(z, x)=\frac{\sum_{r=0}^{N} q(r, x) z^{N-r}}{1-V(x)},
$$

then from $(2.1)$

$$
\begin{gathered}
\frac{\partial}{\partial x} P^{(k, m)}(z, x)+\lambda(z-1) \frac{\partial}{\partial z} P^{(k, m)}(z, x)=0 \\
\frac{\partial}{\partial x} Q(z, x)+\lambda(z-1) \frac{\partial}{\partial z} Q(z, x)=0
\end{gathered}
$$


while the boundary conditions become

$$
\begin{gathered}
Q(z, 0)=z \sum_{k=1}^{N} \int_{0}^{\infty} P^{(k, k)}(z, x) d B(x)+z^{N} \int_{0}^{\infty} q(0, x) \bar{u}(x) d x, \\
P^{(k, m)}(z, 0)=z \int_{0}^{\infty} P^{(k, m-1)}(z, x) d B(x), \quad m=2,3, \ldots, k .
\end{gathered}
$$

Solving (2.9) in the usual way we obtain

$$
Q(z, x)=F\left((z-1) e^{-\lambda x}\right)
$$

where $F$ is an unknown function. Putting $x=0$ we arrive at $F(y)=Q(y+1,0)$ and so finally

$$
Q(z, x)=\sum_{r=0}^{N-1} c_{r}\left[1+(z-1) e^{-\lambda x}\right]^{N-r}
$$

where $\mathrm{c}_{r} \equiv q(r, 0)$. In a similar way

$$
P^{(k, m)}(z, x)=\sum_{r=0}^{N-k+m-1} d_{r}^{(k, m)}\left[1+(z-1) e^{-\lambda x}\right]^{N-k+m-r-1},
$$

with $d_{r}^{(k, m)} \equiv p^{(k, m)}(r, 0)$. Thus to find $P^{(k, m)}(z, x), Q(z, x)$, we have to calculate the quantities $\mathrm{c}_{r}, d_{r}^{(k, m)}$ for all $r, k, m$.

Replacing (2.13) in (2.11), using (2.7) and equating similar powers of $(z-1)$, we arrive at the matrix equation

$$
\mathbf{P}_{k-m}{\underset{\sim}{d}}^{(k, m)}=\mathbf{B}_{k-m}{\underset{\sim}{d}}^{(k, m-1)}
$$

where $\underset{\sim}{d^{(k, m)}}=\left(d_{0}^{(k, m)}, d_{1}^{(k, m)}, \ldots, d_{N+m-k-1}^{(k, m)}\right)^{\prime},(\underset{\sim}{a}$ means the transpose of $\underset{\sim}{a})$ and the $(\mathrm{i}, \mathrm{j})^{t h}$ element of the $(N-n) \times(N-n)$ matrix $\mathbf{P}_{n}$ and of the $(N-n) \times(N-n-1)$ matrix $\mathbf{B}_{n}$ are given respectively by

$$
\begin{gathered}
\left(\mathbf{P}_{n}\right)_{i, j}=\left(\begin{array}{c}
N-n-j \\
N-n-i
\end{array}\right), \\
\left(\mathbf{B}_{n}\right)_{i, j}=\left(\begin{array}{c}
N-n-1-j \\
N-n-1-i
\end{array}\right) B^{*}(\lambda(N-n-1-i))+\left(\begin{array}{c}
N-n-1-j \\
N-n-i
\end{array}\right) B^{*}(\lambda(N-n-i))
\end{gathered}
$$

with $B^{*}(s)$ the LST of $B(t)$. Thus from $(2.14)$

$$
{\underset{\sim}{d}}^{(k, m)}=\prod_{r=0}^{m-2}\left(\mathbf{P}_{k-m+r}^{-1} \mathbf{B}_{k-m+r}\right) \underset{\sim}{d^{(k, 1)}}
$$

Note here that

$$
d_{r}^{(k, 1)} \equiv p^{(k, 1)}(r, 0)= \begin{cases}d_{0}^{(k, 1)} \neq 0 & \text { if } r=0 \\ 0 & \text { if } r \neq 0\end{cases}
$$

and so for all $\mathrm{k}, \underset{\sim}{d^{(k, 1)}}=\left(d_{0}^{(k, 1)}, 0,0, \ldots, 0\right)^{\prime}$. From (2.15) we can obtain all $d_{r}^{(k, m)}$ as functions of the $\mathrm{N}$ unknowns $d_{0}^{(N, 1)}, d_{0}^{(N-1,1)}, \ldots, d_{0}^{(1,1)}$. 
By observing now that a service period starts with $k$ primary customers if and only if the previous vacation period starts with $r$ secondary customers $(r=0,1,2, \ldots, k)$ and during it $(k-r)$ new customers arrive, we obtain, for all $k=1,2, \ldots, N$

$$
d_{0}^{(k, 1)}=\sum_{r=0}^{k} c_{r}\left(\begin{array}{c}
N-r \\
k-r
\end{array}\right) \int_{0}^{\infty}\left(1-e^{-\lambda x}\right)^{k-r} e^{-\lambda(N-k) x} d V(x) .
$$

From (2.16), denoting by $V^{*}(s)$ the LST of $V(t)$, we arrive after manipulations at

$$
d_{0}^{(k, 1)}=\hat{\sim}_{\sim} \stackrel{\sim}{\sim}_{k}^{\prime}
$$

with

$$
\begin{aligned}
& \underset{\sim}{\hat{c}_{k}}=\left(c_{0}, c_{1}, c_{2}, \ldots, c_{k}\right), \quad{\underset{\sim}{\beta}}_{k}=\left(\beta_{k 0}, \beta_{k 1}, \ldots, \beta_{k k}\right), \\
& \beta_{k r}=\left(\begin{array}{c}
N-r \\
N-k
\end{array}\right) \sum_{m=0}^{k-r}(-1)^{m}\left(\begin{array}{c}
k-r \\
m
\end{array}\right) V^{*}(\lambda(N-k+m)), \quad r=0,1, \ldots, k .
\end{aligned}
$$

Note here that we can also obtain relation (2.17) directly from (2.4) using (2.12) and the definition (2.7). In a similar way, using (2.12) and (2.13) in (2.10), we arrive at

$$
c_{r}=\underset{\sim}{\alpha} \mathbf{D}_{\sim}{ }_{\sim}^{\prime}, \quad r=1,2, \ldots, N-1
$$

where

$$
\begin{array}{ll}
\underset{\sim}{\alpha_{r}}=\left(\alpha_{r 0}, \alpha_{r 1}, \ldots, \alpha_{r r}, 0, \ldots, 0\right), & \underset{\sim}{1}=(1,1, \ldots, 1), \\
\alpha_{r m}=\left(\begin{array}{c}
N-1-m \\
r-m
\end{array}\right) \sum_{k=0}^{r-m}(-1)^{k}\left(\begin{array}{c}
r-m \\
k
\end{array}\right) B^{*}(\lambda(N-r+k-1)),
\end{array}
$$

with $m=0,1, \ldots r$ and the $N \times N$ matrix $\mathbf{D}$ has $\underset{\sim}{d^{(k, k)}}$ as its $k^{t h}$ column, $k=1,2, \ldots, N$.

Using finally relations (2.15) and (2.17) in (2.19), and after manipulations, we obtain

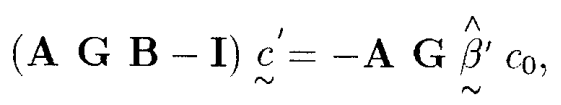

where $\underset{\sim}{c}=\left(c_{1}, c_{2}, \ldots, c_{N-1}\right), \underset{\sim}{\hat{\beta}}=\left(\beta_{10}, \beta_{20}, \ldots, \beta_{N 0}\right)$ and $\mathbf{A}$ is the $(N-1) \times N$ matrix with elements $(\mathbf{A})_{i j}=\alpha_{i j-1}$ defined by $(2.20), \mathbf{B}$ is the $N \times(N-1)$ matrix with $(\mathbf{B})_{i j}=\beta_{i j}$

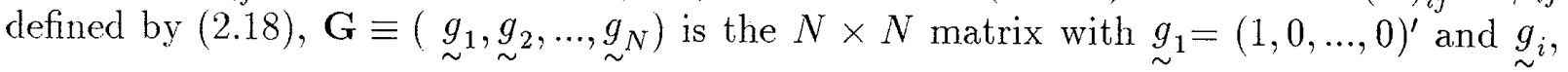
$i=2,3, \ldots, N$, equal to the first column of the matrix $\prod_{r=0}^{i-2}\left(\mathbf{P}_{r}^{-1} \mathbf{B}_{r}\right)$ defined in (2.15).

Putting now $c_{0}=1$ in (2.21), we obtain $c_{r}$ for all $r=1,2, \ldots, N-1\left(c_{N}=0\right)$ and so, from $(2.17),(2.15), d_{r}^{(k, m)}$ can also be obtained for all $r, k, m$. Using finally these quantities we calculate

$R=\int_{0}^{\infty}\left[(1-V(x)) Q(1, x)+(1-B(x)) \sum_{k, m} \mathrm{P}^{(k, m)}(1, x)\right] d x=\bar{v} \sum_{r=0}^{N-1} c_{r}+\bar{b} \sum_{k, m} \sum_{r=0}^{N-k+m-1} d_{r}^{(k, m)}$.

Obviously, $\mathrm{R}$ must be equal to one and so it is easy to understand (from the form of (2.21), $(2.17)$ and $(2.15))$ that, to get the final values of $c_{r}, d_{r}^{(k, m)}$ for all $r, k, m$, we have to divide the previously obtained quantities (for $c_{0}=1$ ) by R. Finally, by comparing equations (2.12), (2.13) with the definitions (2.7) and equating similar powers of $(z-1)$, we can easily obtain the exact probabilities $q(r, x)$ and $p^{(k, m)}(r, x)$ as functions of the known quantities $c_{r}, d_{r}^{(k, m)}$. 


\section{Waiting time process (FCFS discipline)}

Consider the probabilities

$$
\begin{gathered}
p_{r \in m}^{(k, m)}(r, y) d y=\lim _{t \rightarrow \infty} \operatorname{Pr}\left[n(t)=k, A_{n(t)}=m, m(t)=r, y<\bar{S}(t) \leq y+d y\right], \\
q_{r e m}(r, y) d y=\lim _{t \rightarrow \infty} \operatorname{Pr}\left[n(t)=0, m(t)=r, y<\bar{V}_{0}(t) \leq y+d y\right],
\end{gathered}
$$

where $\bar{S}(t), \bar{V}_{0}(t)$ is now the remaining service or vacation time, respectively. Then

$$
p_{r e m}^{(k, m)}(r, y)=\int_{0}^{\infty} p^{(k, m)}(r, x) \frac{b(x+y)}{1-B(x)} d x, \quad q_{r e m}(r, y)=\int_{0}^{\infty} q(r, x) \frac{v(x+y)}{1-V(x)} d x,
$$

and using (2.12), (2.13),

$$
Q_{r e m}(z, s)=\sum_{r=0}^{N} c_{r} \sum_{j=0}^{N-r}\left(\begin{array}{c}
N-r \\
j
\end{array}\right) \frac{V^{*}(s)-V^{*}(j \lambda)}{\lambda j-s}(z-1)^{j},
$$

$$
P_{r \in m}^{(k, m)}(z, s)=\sum_{r=0}^{N-k+m-1} d_{r}^{(k, m)} \sum_{j=0}^{N-k+m-r-1}\left(\begin{array}{c}
N-k+m-r-1 \\
j
\end{array}\right) \frac{B^{*}(s)-B^{*}(j \lambda)}{\lambda j-s}(z-1)^{j},
$$

where now

$$
\begin{gathered}
Q_{r e m}(z, s)=\sum_{r=0}^{N} z^{N-r} \int_{0}^{\infty} e^{-s y} q_{r e m}(r, y) d y \\
P_{r e m}^{(k, m)}(z, s)=\sum_{r=0}^{N-k+m-1} z^{N-k+m-r-1} \int_{0}^{\infty} e^{-s y} p_{r e m}^{(k, m)}(r, y) d y .
\end{gathered}
$$

We have to point out here that, as Takagi [2] also observed, the state of the system seen by an arriving customer is different from the state at an arbitrary time and so, for the probabilities, at the arrival epochs, we have

$$
p_{a r r}^{(k, m)}(r, y)=\frac{(N-k+m-r-1) \lambda}{\gamma} p_{r e m}^{(k, m)}(r, y), \quad q_{a r r}(r, y)=\frac{(N-r) \lambda}{\gamma} q_{r e m}(r, y),
$$

where $\gamma=\left(1-B^{*}(\lambda)\right) \varphi_{1}+\left(1-V^{*}(\lambda)\right) \psi_{1}$ and for $i=1,2$

$$
\psi_{i}=\sum_{r=0}^{N}\left(\begin{array}{c}
N-r \\
i
\end{array}\right) c_{r}, \quad \varphi_{i}=\sum_{k=1}^{N} \sum_{m=1}^{k} \sum_{r=0}^{N-k+m-1}\left(\begin{array}{c}
N-k+m-r-1 \\
i
\end{array}\right) d_{r}^{(k, m)}
$$

Let now $W$ be the steady state waiting time in queue (excluding service) of an arbitrary customer and let $W^{*}(s)$ be the LST of the distribution function of W. Then we can write

$$
\begin{aligned}
& \gamma W^{*}(s)=\sum_{k=0}^{N}\left[B^{*}(s)\right]^{k}(N-k) \lambda \int_{0}^{\infty} e^{-s y} q_{r e m}(k, y) d y \\
& +V^{*}(s) \sum_{k=1}^{N} \sum_{m=1}^{k} \sum_{r=0}^{N-k+m-1}\left[B^{*}(s)\right]^{k+r-m}(N-k+m-r-1) \lambda \int_{0}^{\infty} e^{-s y} p_{r e m}^{(k, m)}(r, y) d y .
\end{aligned}
$$

From (3.5), using (3.2) we obtain

$$
\begin{aligned}
\gamma W^{*}(s) & =\left.\lambda\left(B^{*}(s)\right)^{N-1} \frac{\partial}{\partial z} Q_{r e m}(z, s)\right|_{z=\left(B^{*}(s)\right)^{-1}} \\
& +\left.\lambda V^{*}(s)\left(B^{*}(s)\right)^{N-2} \sum_{k=1}^{N} \sum_{m=1}^{k} \frac{\partial}{\partial z} P_{r e m}^{(k, m)}(z, s)\right|_{z=\left(B^{*}(s)\right)^{-1}}
\end{aligned}
$$


and so from $(3.1)$

$$
\begin{aligned}
\gamma W^{*}(s) & =\lambda \sum_{r=0}^{N} c_{r} \sum_{j=0}^{N-r} j\left(\begin{array}{c}
N-r \\
j
\end{array}\right) \frac{V^{*}(s)-V^{*}(j \lambda)}{\lambda j-s}\left(1-B^{*}(s)\right)^{j-1}\left(B^{*}(s)\right)^{N-j} \\
& +\lambda V^{*}(s) \sum_{k=1}^{N} \sum_{m=1}^{k} \sum_{r=0}^{N-k+m-1} d_{r}^{(k, m)} \sum_{j=0}^{N-k+m-r-1} j\left(\begin{array}{c}
N-k+m-r-1 \\
j
\end{array}\right) \\
& \times \frac{B^{*}(s)-B^{*}(j \lambda)}{\lambda j-s}\left(1-B^{*}(s)\right)^{j-1}\left(B^{*}(s)\right)^{N-j-1}
\end{aligned}
$$

and so $W^{*}(s)$ is completely known. Finally, by differentiating (3.6), we obtain the mean waiting time $E(W)$ as

$E(W)=\bar{v}+(N-1) \bar{b}-\frac{1}{\lambda}+\frac{\bar{v}}{\gamma} \psi_{1} V^{*}(\lambda)+\frac{\bar{b}}{\gamma}\left[\varphi_{1} B^{*}(\lambda)-\psi_{2}\left(1-V^{*}(2 \lambda)\right)-\varphi_{2}\left(1-B^{*}(2 \lambda)\right)\right]$

where $\psi_{i}, \varphi_{i}, i=1,2$, are given by (3.4).

\section{Numerical Results-Conclusions}

To observe the way in which the customers' mean waiting time is affected when we vary the values of the parameters, and to compare our model with the corresponding models with exhaustive service and with infinite customer population, we present here Table 1.

To construct the Table we denoted by $W_{e}^{(N)}$ and $W_{g}^{(N)}$ the mean waiting times in the model of customer population $\mathrm{N}$ with exhaustive and gated service respectively, and assumed that the service and the vacation times follow exponential distributions with parameters $\frac{1}{\bar{b}}$ and $\frac{1}{\bar{v}}$ respectively. We used $\bar{b}=0.5$.

Table 1 depicts values of $W^{(\cdot)}$ when we increase the offered load $\rho=N \lambda \bar{b}$, and vary the mean vacation time $\bar{v}$. To obtain values for $W_{e}^{(N)}$ we have used formula (2.17) in Takagi [2] while for the values of $W_{e}^{(\infty)}, W_{g}^{(\infty)}$ we used, with $\rho=\lambda \bar{b}$ now, formulas (2.14a) and (5.24a) of chapter 2 in Takagi [1].

One can observe in the Table that, as it is expected, the values of $W_{g}^{(.)}$are, for all systems, greater than the corresponding values of $W_{e}^{(.)}$. This difference becomes more apparent for large values of $\rho$ and particularly for large $\bar{v}$. Thus, in case of $\bar{v}=5$, we have $W_{e}^{(4)}=4.614$, $W_{g}^{(4)}=5.637$ for $\rho=0.4$, while for $\rho=5$ the corresponding values are $W_{e}^{(4)}=1.678$ and $W_{g}^{(4)}=6.266$.

One can also observe in Table 1 the way in which the models are affected from variations in the vacation periods. An interesting observation here is that in the case of gated service and for all $\bar{v}$ the value of $W_{g}^{(.)}$is always increasing with $\rho$, a phenomenon that we cannot always observe in the case of exhaustive service. This behavior is a consequence of the vacation principle and is more apparent for large $\bar{v}$. In systems with gated service the customers' waiting time always contains a vacation period or a part of a vacation period and so $W_{g}^{(.)}$is always affected from $\bar{v}$. For systems with exhaustive service, when $\rho$ increases the duration of the busy period increases and the opportunity for a vacation becomes now rare. Thus in an exhaustive service system with large $\bar{v}$, the effect of the vacation, which is large a.t the beginning (when $\rho$ is small), is reduced when $\rho$ increases and it results in smaller values of the mean waiting times. Of course, beyond a certain value of $\rho$, this effect is wiped out, and the waiting time starts again to increase with $\rho$ (see for example $W_{e}^{(12)}$ for $\bar{v}=5$ ). An observation supporting the previous explanation is that for a very large $\rho, \rho=5$ for example, the mean waiting times $W_{\epsilon}^{(.)}$become almost identical for all $\bar{v}$ (the vacation does not affect the models at all), while for $\rho=5$ the differences between the mean waiting 
times $W_{g}^{(.)}$are almost equal to the differences between the corresponding mean vacations (the models are fully dependent on the vacation periods).

Finally one can observe in Table 1 , how the mean waiting times increase when we increase the population size $\mathrm{N}$.

\begin{tabular}{|c|l|r|r|r|r|r|r|r|}
\hline $\bar{v} \backslash \rho$ & & $\mathbf{0 . 4}$ & $\mathbf{0 . 6}$ & $\mathbf{0 . 8}$ & $\mathbf{1}$ & $\mathbf{2}$ & $\mathbf{4}$ & $\mathbf{5}$ \\
\hline \multirow{5}{*}{$\mathbf{1}$} & $W_{e}^{(4)}$ & 1.094 & 1.115 & 1.127 & 1.135 & 1.203 & 1.295 & 1.326 \\
& $W_{g}^{(4)}$ & 1.373 & 1.507 & 1.616 & 1.705 & 1.976 & 2.191 & 2.244 \\
& $W_{e}^{(8)}$ & 1.167 & 1.247 & 1.328 & 1.411 & 1.859 & 2.523 & 2.707 \\
& $W_{g}^{(8)}$ & 1.563 & 1.845 & 2.106 & 2.340 & 3.117 & 3.726 & 3.867 \\
& $W_{e}^{(12)}$ & 1.205 & 1.330 & 1.476 & 1.645 & 2.695 & 4.008 & 4.300 \\
& $W_{g}^{(12)}$ & 1.661 & 2.053 & 2.453 & 2.836 & 4.205 & 5.281 & 5.522 \\
& $W_{e}^{(\infty)}$ & 1.333 & 1.750 & 3.000 & $\infty$ & $\infty$ & $\infty$ & $\infty$ \\
& $W_{g}^{(\infty)}$ & 2.000 & 3.250 & 7.000 & $\infty$ & $\infty$ & $\infty$ & $\infty$ \\
\hline & $W_{e}^{(4)}$ & 1.991 & 1.939 & 1.879 & 1.819 & 1.574 & 1.371 & 1.342 \\
& $W_{g}^{(4)}$ & 2.485 & 2.619 & 2.719 & 2.797 & 3.027 & 3.210 & 3.257 \\
& $W_{e}^{(8)}$ & 2.072 & 2.062 & 2.042 & 2.022 & 2.074 & 2.540 & 2.714 \\
$\mathbf{2}$ & $W_{g}^{(8)}$ & 2.788 & 3.103 & 3.366 & 3.586 & 4.259 & 4.777 & 4.902 \\
& $W_{e}^{(12)}$ & 2.124 & 2.156 & 2.188 & 2.232 & 2.812 & 4.004 & 4.301 \\
& $W_{g}^{(12)}$ & 2.960 & 3.426 & 3.851 & 4.227 & 5.445 & 6.379 & 6.591 \\
& $W_{e}^{(\infty)}$ & 2.333 & 2.750 & 4.000 & $\infty$ & $\infty$ & $\infty$ & $\infty$ \\
& $W_{g}^{(\infty)}$ & 3.667 & 5.750 & 12.000 & $\infty$ & $\infty$ & $\infty$ & $\infty$ \\
\hline & $W_{e}^{(4)}$ & 4.614 & 4.340 & 4.071 & 3.816 & 2.812 & 1.871 & 1.678 \\
& $W_{g}^{(4)}$ & 5.637 & 5.748 & 5.827 & 5.887 & 6.068 & 6.224 & 6.266 \\
& $W_{e}^{(8)}$ & 4.684 & 4.382 & 4.064 & 3.753 & 2.703 & 2.599 & 2.733 \\
$\mathbf{5}$ & $W_{g}^{(8)}$ & 6.168 & 6.474 & 6.701 & 6.879 & 7.399 & 7.821 & 7.930 \\
& $W_{e}^{(12)}$ & 4.766 & 4.486 & 4.172 & 3.862 & 3.154 & 4.010 & 4.302 \\
& $W_{g}^{(12)}$ & 6.524 & 7.033 & 7.435 & 7.760 & 8.735 & 9.481 & 9.658 \\
& $W_{e}^{(\infty)}$ & 5.333 & 5.750 & 7.000 & $\infty$ & $\infty$ & $\infty$ & $\infty$ \\
& $W_{g}^{(\infty)}$ & 8.667 & 13.250 & 27.000 & $\infty$ & $\infty$ & $\infty$ & $\infty$ \\
\hline
\end{tabular}

Table 1: Values of the mean waiting time for $\bar{b}=0.5$.

\section{Acknowledgement}

We would like to thank Dr P. Yalamov from Technical University of Russe, Bulgaria, for his help with the numerical computations.

\section{References}

[1] Takagi, H., Queueing Analysis, Foundation of Performance Evaluation, Vol. 1: Vacation and Priority Systems, Part 1, North-Holland (1991), Amsterdam.

[2] Takagi, H., Analysis of an $\mathrm{M} / \mathrm{G} / 1 / \mathrm{N}$ queue with multiple server vacations, and its application to a polling model, J. Oper. Res. Soc. Japan, Vol 35 (1992), 300-315.

[3] Takine, T. and Hasegawa, T., On the M/G/1 queue with multiple vacations and gated service discipline, J. Oper. Res. Soc. Japan, Vol 35 (1992), 217-235.

\section{C.LANGARIS and A.KATSAROS \\ University of Ioannina \\ Department of Mathematics \\ 45110 Ioannina, Greece}

